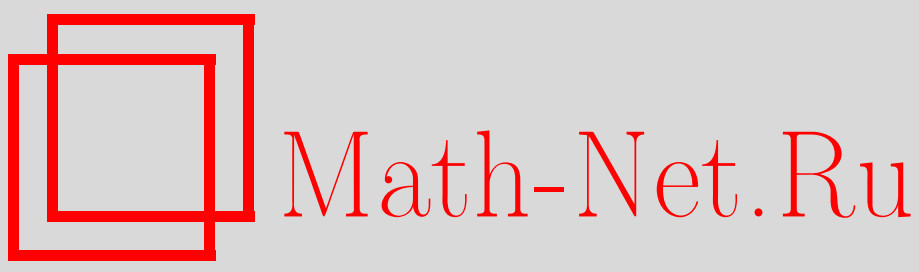

А. Г. Багдасарян, Об интерполяционных свойствах некоторых квазилинеаризуемых пар, Матем. сб., 1998, том 189, номер 2, 73-80

DOI: https://doi.org/10.4213/sm291

Использование Общероссийского математического портала Math-Net.Ru подразумевает, что вы прочитали и согласны с пользовательским соглашением http://www . mathnet.ru/rus/agreement

Параметры загрузки:

IP: 54.224 .187 .69

26 апреля 2023 г., $12: 07: 18$ 
УДК 517.5

\author{
А.Г. Багдасарян
}

\title{
Об интерполяционных свойствах некоторых квазилинеаризуемых пар
}

\footnotetext{
Для квазилинеаризуемой пары рефлексивных пространств $\left\{A_{0}, A_{1}\right\}$ исследуются интерполяционные свойства производной пары $\left\{A_{0}+A_{1}, A_{0} \cap A_{1}\right\}$. Доказьвается формула вещественной интерполяции, связывающая интерполяционные пространства пар $\left\{A_{0}, A_{1}\right\}$ и $\left\{A_{0}+A_{1}, A_{0} \cap A_{1}\right\}$. В частности, оказывается, что при $\theta=\frac{1}{2}$ соответствующие интерполяционные пространства совпадают. Полученные результаты применяются к обобщенным пространствам типа Никольского-Бесова (предварительно доказывается квазилинеаризуемость пары $\left\{B_{p, q}^{1}\left(\mu_{0}\right), B_{p, q}^{1}\left(\mu_{1}\right)\right\}$ таких пространств).

Библиография: 12 названий.
}

В работе исследуются некоторые вопросы теории вешественной интерполяции и указываются некоторые их применения в теории пространств функций обобшенной гладкости типа Никольского-Бесова.

Для всякого интерполяционного пространства $A$ относительно пары $\vec{A}=$ $\left\{A_{0}, A_{1}\right\}$ банаховых пространств по определению $\Delta \subset A \subset \Sigma$, где $\Delta=\Delta(\vec{A})=$ $A_{0} \cap A_{1}, \Sigma=\Sigma(\vec{A})=A_{0}+A_{1}$ (см. определение 1$)$. В связи с этим представляет интерес исследование интерполяционных свойств пары $\{\Sigma, \Delta\}$ (исследования такого рода приводились еше в шестидесятые годы [1]).

В настоящей работе мы намереваемся установить связь между интерполяционными пространствами пар $\left\{A_{0}, A_{1}\right\}$ и $\{\Sigma, \Delta\}$. Для так называемых квазилинеаризуемых пар (см. определение 2) удается установить явную формулу, связывающую интерполяционные пространства относительно указанных пар, полученные методом вещественной интерполяции. Многие вопросы теории вешественной интерполяции находят свое окончательное решение для квазилинеаризуемых пар (см. [2]). И хотя Петре, которьй ввел это понятие, в [2] показал, что требование квазилинеаризуемости несколько ограничительно (в частности, пара $\left\{L_{p_{0}}, L_{p_{1}}\right\}$ при $p_{0} \neq p_{1}$, вообще говоря, не квазилинеаризуема), тем не менее, мы доказываем, что многие интересные пары пространств функций обобщенной гладкости (пространства типа Соболева-Лиувилля, Никольского-Бесова) обладают свойством квазилинеаризуемости. В этой связи результаты настоящей работы находят свое применение во многих вопросах теории интерполяции и теории вложений для пространств функций обобщенной гладкости.

\section{§ 1. О квазилинеаризуемости некоторых интерполяционных пар}

ОПРЕДЕЛЕНИЕ 1. Пусть $A_{0}$ и $A_{1}$ два банаховых пространства, линейно и непрерывно вложенных в линейное топологическое пространство $T$. Пару $\vec{A}=\left\{A_{0}, A_{1}\right\}$ таких пространств будем называть интерполяционной парой. Положим

(C) А.Г. БАГДАСАрян 
a) $A_{0}+A_{1}=\left\{a \in T: a=a_{0}+a_{1}, a_{j} \in A_{j}, j=0,1\right\}$,

$$
\|a\|_{A_{0}+A_{1}}=\inf _{\substack{a=a_{0}+a_{1} \\ a_{j} \in A_{j}}}\left(\left\|a_{0}\right\|_{A_{0}}+\left\|a_{1}\right\|_{A_{1}}\right)
$$

б) $A_{0} \cap A_{1}=\left\{a \in T:\|a\|_{A_{0} \cap A_{1}}=\|a\|_{A_{0}}+\|a\|_{A_{1}}<\infty\right\}$.

Для краткости записи обозначим

$$
A_{0}+A_{1}=\Sigma(\vec{A}), \quad A_{0} \cap A_{1}=\Delta(\vec{A}) .
$$

ОПРЕДЕЛЕНИЕ 2. Интерполяционная пара $\left\{A_{0}, A_{1}\right\}$ назьвается квазилинеаризуемой, если существует пара операторов $\left\{V_{0}(t), V_{1}(t)\right\}, V_{j}(t) \in L\left(\Sigma, A_{j}\right), j=0,1$, $0<t<\infty$, такая, что

$$
\begin{array}{cl}
V_{0}(t)+V_{1}(t)=E \text { в } & \Sigma(\vec{A}), \\
\left\|V_{0}(t) a\right\|_{A_{0}} \leqslant c\|a\|_{A_{0}}, & a \in A_{0}, \\
\left\|V_{0}(t) a\right\|_{A_{0}} \leqslant c t\|a\|_{A_{1}}, & a \in A_{1}, \\
\left\|V_{1}(t) a\right\|_{A_{1}} \leqslant \frac{c}{t}\|a\|_{A_{0}}, & a \in A_{0}, \\
\left\|V_{1}(t) a\right\|_{A_{1}} \leqslant c\|a\|_{A_{1}}, & a \in A_{1} .
\end{array}
$$

Нетрудно убедиться, что соотношения (1), (2), (5) влекут за собой оценки

$$
\begin{aligned}
& \left\|V_{0}(t) a\right\|_{A_{1}} \leqslant c\|a\|_{A_{1}}, \quad a \in A_{1}, \\
& \left\|V_{1}(t) a\right\|_{A_{0}} \leqslant c\|a\|_{A_{0}}, \quad a \in A_{0} .
\end{aligned}
$$

ТеОРема 1. Пусть $\left\{A_{0}, A_{1}\right\}$ - квазилинеаризуемая интерполяционная пара с операторами $\left\{V_{0}(t), V_{1}(t)\right\}$. Тогда пара $\left\{\Sigma, A_{1}\right\}$ тоже квазилинеаризуема $c$ операторами $\left\{W_{0}(t), W_{1}(t)\right\}$, əде

$$
W_{1}(t)=\frac{V_{1}(t)}{t \max \{1 ; 1 / t\}}, \quad W_{0}(t)=E-W_{1}(t) .
$$

ДокАЗАТЕЛЬСтво. Для $a=a_{0}+a_{1}, a_{j} \in A_{j}, j=0,1$, имеем

$$
\begin{aligned}
\left\|W_{0}(t) a\right\|_{\Sigma} & \leqslant\|a\|_{\Sigma}+\left\|V_{1}(t) a\right\|_{\Sigma} \leqslant\|a\|_{\Sigma}+c\left(\left\|V_{1}(t) a_{0}\right\|_{A_{0}}+\left\|V_{1}(t) a_{1}\right\|_{A_{1}}\right) \\
& \leqslant\|a\|_{\Sigma}+c^{\prime}\left(\left\|a_{0}\right\|_{A_{0}}+\left\|a_{1}\right\|_{A_{1}}\right) .
\end{aligned}
$$

Использовали соотношения (5), (7), вложения $A_{j} \subset \Sigma, j=0,1$, и неравенство $t \max \{1 ; 1 / t\} \geqslant 1$. Выбирая нижнюю грань по всем разложениям, приходим $\mathrm{K}$ аналогу оценки (2) для пары $\left\{\Sigma, A_{1}\right\}$. Для $a \in A_{1}, t \geqslant 1$ имеем

$$
\left\|W_{0}(t) a\right\|_{\Sigma} \leqslant c\left(\|a\|_{A_{1}}+\left\|V_{1}(t) a\right\|_{A_{1}}\right) \leqslant c^{\prime} t\|a\|_{A_{1}} .
$$

Использовали (5) и вложение $A_{1} \subset \Sigma$. 
Пусть теперь $0<t \leqslant 1$. Из (1), (3) и вложения $A_{0} \subset \Sigma$ имеем

$$
\left\|W_{0}(t) a\right\|_{\Sigma}=\left\|V_{0}(t) a\right\|_{\Sigma} \leqslant c\left\|V_{0}(t) a\right\|_{A_{0}} \leqslant c^{\prime} t\|a\|_{A_{1}} .
$$

Оценки (8), (9) доказывают аналог оценки (3). При $a=a_{0}+a_{1}, a_{j} \in A_{j}, j=0,1$, имеем

$$
\begin{aligned}
\left\|W_{1}(t) a\right\|_{A_{1}} & \leqslant \frac{1}{t \max \{1 ; 1 / t\}}\left(\left\|V_{1}(t) a_{0}\right\|_{A_{1}}+\left\|V_{1}(t) a_{1}\right\|_{A_{1}}\right) \\
& \leqslant \frac{c}{t \max \{1 ; 1 / t\}}\left(\frac{1}{t}\left\|a_{0}\right\|_{A_{0}}+\left\|a_{1}\right\|_{A_{1}}\right) \leqslant \frac{c}{t}\left(\left\|a_{0}\right\|_{A_{0}}+\left\|a_{1}\right\|_{A_{1}}\right) .
\end{aligned}
$$

Выбирая нижнюю грань, приходим к аналогу оценки (4). Наконец, для $a \in A_{1}$ (см. (5)) имеем

$$
\left\|W_{1}(t) a\right\|_{A_{1}} \leqslant\left\|V_{1}(t) a\right\|_{A_{1}} \leqslant c\|a\|_{A_{1}} .
$$

Теорема доказана.

ЗАмечАнИЕ. В предположениях теоремы 1 нетрудно убедиться, что пара $\left\{A_{1}, A_{0}\right\}$ тоже квазилинеаризуема с операторами $\left\{V_{1}(1 / t), V_{0}(1 / t)\right\}$. Тогда из теоремы 1 следует квазилинеаризуемость пары $\left\{\Sigma, A_{0}\right\}$.

Теорема 2. Пусть $\left\{A_{0}, A_{1}\right\}$ - квазилинеаризуемая пара рефлексивных пространств с операторами $\left\{V_{0}(t), V_{1}(t)\right\}$. Тогда пара $\left\{A_{0}^{*}, A_{1}^{*}\right\}$ тоже квазилинеаризуема с операторами $\left\{V_{1}^{*}(1 / t), V_{0}^{*}(1 / t)\right\}$, где $A_{j}^{*}$ - сопряжсенное пространство $к A_{j}$ и $V_{j}^{*}(t)$ - сопряженный оператор $\kappa V_{j}(t), j=0,1$.

ДокАЗАТЕЛЬСТво. Из (7) для $a \in A_{0}^{*}$ имеем

$$
\left\|V_{1}^{*}\left(\frac{1}{t}\right) a\right\|_{A_{0}^{*}}=\sup _{\varphi \neq 0} \frac{\left|\left\langle a, V_{1}(1 / t) \varphi\right\rangle\right|}{\|\varphi\|_{A_{0}}} \leqslant c\|a\|_{A_{0}^{*}} .
$$

Аналогично, с использованием (6) доказываем аналог оценки (5). Нетрудно убедиться, что остальные оценки тоже имеют место. Теорема доказана.

\section{§ 2. Интерполяция суммы и пересечение}

ТЕОРема 3. Пусть квазилинеаризуемая пара рефлексивных пространств $\left\{A_{0}, A_{1}\right\}$ такова, что $\Delta(\vec{A})$ плотно в $A_{j}, A_{0}^{*} \cap A_{1}^{*} \equiv \Delta\left(A^{*}\right)$ плотно в $A_{j}^{*}$, $j=0,1$. Тогда пара $\{\Sigma(\vec{A}), \Delta(\vec{A})\}$ тоже квазилинеаризуема и

$$
(\Sigma(\vec{A}), \Delta(\vec{A}))_{1 / 2, q}=\left(A_{0}, A_{1}\right)_{1 / 2, q}, \quad 1<q<\infty .
$$

ДоКАЗАТЕЛЬСТво. Из рефлексивности пространств $A_{j}, j=0,1$, теоремы 2.7.1 из [3] (поскольку $\Delta(\vec{A})$ плотно в $A_{j}$ ) имеем

$$
\Delta(\vec{A}) \supset\left(A_{0}^{*}+A_{1}^{*}\right)^{*}=\left(A_{0} \cap A_{1}\right)^{* *}=\Delta^{* *}(\vec{A}) .
$$

То есть пространство $\Delta(\vec{A})$ реффлексивно. Поскольку $\Delta\left(\vec{A}^{*}\right)$ плотно в $A_{j}^{*}, j=0,1$, то теорема 2.7.1 из [3] применима и к паре $\left\{A_{0}^{*}, A_{1}^{*}\right\}$. Тогда

$$
\left(A_{0}+A_{1}\right)^{* *}=\left(A_{0}^{*} \cap A_{1}^{*}\right)^{*}=A_{0}^{* *}+A_{1}^{* *}=A_{0}+A_{1} .
$$


То есть $\Sigma(\vec{A})$ - тоже рефилексивно.

Далее, ясно, что для $0<\theta<1,1<q<\infty$

$$
\begin{aligned}
\Delta\left(\vec{A}^{*}\right) & \subset\left(A_{0}, A_{1}\right)_{\theta, q}^{*} \cap\left(A_{1}, A_{0}\right)_{\theta, q}^{*}, \\
\Delta(\vec{A}) & \subset\left(A_{0}, A_{1}\right)_{\theta, q} \cap\left(A_{1}, A_{0}\right)_{\theta, q} .
\end{aligned}
$$

Тогда из теорем 3.4.2б) и 3.7.1 из [3] следует, что

$$
\begin{aligned}
& \left(A_{0}, A_{1}\right)_{\theta, q} \cap\left(A_{1}, A_{0}\right)_{\theta, q} \quad \text { плотно в }\left(A_{0}, A_{1}\right)_{\theta, q} \quad \text { и }\left(A_{1}, A_{0}\right)_{\theta, q} \text {, } \\
& \left(A_{0}, A_{1}\right)_{\theta, q}^{*} \cap\left(A_{1}, A_{0}\right)_{\theta, q}^{*} \quad \text { плотно в }\left(A_{0}, A_{1}\right)_{\theta, q}^{*} \quad \text { и }\left(A_{1}, A_{0}\right)_{\theta, q}^{*} \text {. }
\end{aligned}
$$

Поскольку (см. [4, замечание 1.11.3]) из рефилексивности $A_{j}, j=0,1$, учитьвая соображения плотности, следует рефилексивность пространств $\left(A_{0}, A_{1}\right)_{\theta, q}, 0<\theta<1$, $1<q<\infty$, то предыдущие рассуждения показывают, что рефилексивно пространство $\left(A_{0}, A_{1}\right)_{\theta, q}+\left(A_{1}, A_{0}\right)_{\theta, q}$. Тогда из теоремы 2.7.1 из [3] следует, что

$$
\left(A_{0}, A_{1}\right)_{\theta, q}+\left(A_{1}, A_{0}\right)_{\theta, q}=\left(\left(A_{0}, A_{1}\right)_{\theta, q}^{*} \cap\left(A_{1}, A_{0}\right)_{\theta, q}^{*}\right)^{*} .
$$

Используя доказательство теоремы 1.12.1 из [4] (учитывая теорему 1, замечание 1 , явньй вид операторов $W_{j}(t)$ и оценки $\left.(6),(7)\right)$, убеждаемся в квазилинеаризуемости пары $\{\Sigma(\vec{A}), \Delta(\vec{A})\}$ (соответствующие операторы выписываются в теореме 1.12.1 из [4]). Отметим, что при этом мы не пользуемся условием коммутативности нулевых операторов.

Далее, из теоремы 1 , явного вида операторов $W_{j}(t)$ и $(7)$ следует, что выполнены условия предложения 1.1 из [5]. На основании этого предложения имеем

$$
(\Sigma(\vec{A}), \Delta(\vec{A}))_{\theta, q}=\left(\Sigma(\vec{A}), A_{0}\right)_{\theta, q} \cap\left(\Sigma(\vec{A}), A_{1}\right)_{\theta, q} .
$$

На основании теоремы 2 и соответствующих рассуждений аналогичная формула имеет место и для пары $\left\{\Sigma\left(\vec{A}^{*}\right), \Delta\left(\vec{A}^{*}\right)\right\}$. Тогда из (12) при $0<\theta<1,1<q<\infty$ имеем

$$
\begin{aligned}
&(\Sigma(\vec{A}), \Delta(\vec{A}))_{\theta, q} \supset\left(A_{1}, A_{0}\right)_{\theta, q} \cap\left(A_{0}, A_{1}\right)_{\theta, q}, \\
&\left(\Sigma\left(\vec{A}^{*}\right), \Delta\left(\vec{A}^{*}\right)\right)_{\theta, q} \supset\left(A_{1}^{*}, A_{0}^{*}\right)_{\theta, q} \cap\left(A_{0}^{*}, A_{1}^{*}\right)_{\theta, q} .
\end{aligned}
$$

Далее, из (11), (14), теорем 3.7.1, 2.7.1 из [3] имеем $\left(1 / q+1 / q^{\prime}=1\right)$

$$
\begin{aligned}
\left(A_{0}, A_{1}\right)_{\theta, q}+\left(A_{1}, A_{0}\right)_{\theta, q} & =\left(\left(A_{0}^{*}, A_{1}^{*}\right)_{\theta, q^{\prime}} \cap\left(A_{1}^{*}, A_{0}^{*}\right)_{\theta, q^{\prime}}\right)^{*} \\
& \supset\left(\Sigma\left(\vec{A}^{*}\right), \Delta\left(\vec{A}^{*}\right)\right)_{\theta, q^{\prime}}^{*}=(\Delta(\vec{A}), \Sigma(\vec{A}))_{\theta, q} .
\end{aligned}
$$

Формулы (13), (15) при $\theta=\frac{1}{2}$ приводят нас к (10). Теорема доказана.

ТЕОрема 4. В предполодсениях теоремы 3 имеют место соотношения

$$
\begin{array}{ll}
(\Sigma(\vec{A}), \Delta(\vec{A}))_{\theta, q}=\left(A_{0}, A_{1}\right)_{\theta, q} \cap\left(A_{1}, A_{0}\right)_{\theta, q}, & \frac{1}{2} \leqslant \theta<1, \\
(\Sigma(\vec{A}), \Delta(\vec{A}))_{\theta, q}=\left(A_{0}, A_{1}\right)_{\theta, q}+\left(A_{1}, A_{0}\right)_{\theta, q}, & 0<\theta \leqslant \frac{1}{2} .
\end{array}
$$


ДокАзАТЕЛьство. Согласно [6] (см. также замечание 1.3.2/2 из [4]) имеем

$$
(\Sigma, \Delta)_{1, \infty}=\Delta, \quad(\Sigma, \Delta)_{0, \infty}=\Sigma .
$$

Тогда из теоремы реитерации (см. [3, теорема 3.5.3], а также [3, определение 3.5.3 и рассуждения после определения]) имеем

$$
\begin{aligned}
& \left((\Sigma, \Delta)_{1, \infty},(\Sigma, \Delta)_{1 / 2, r}\right)_{\eta, q}=(\Sigma, \Delta)_{\theta, q}, \\
& 1 \leqslant q \leqslant \infty, \quad 0<\eta<1, \quad \theta=1-\frac{\eta}{2} .
\end{aligned}
$$

Аналоги формул (18), (19) по тем же соображениям верны и для пары $\left\{A_{0}, A_{1}\right\}$. Для произвольного $\theta \in\left(\frac{1}{2}, 1\right)$ найдем соответствуюшее $\eta$. Воспользовавшись (18), (19) и теоремой 3, получим

$$
\begin{aligned}
(\Sigma, \Delta)_{\theta, q} & =\left(\Delta,\left(A_{0}, A_{1}\right)_{1 / 2, r}\right)_{\eta, q} \subset\left(A_{0},\left(A_{0}, A_{1}\right)_{1 / 2, r}\right) \cap\left(A_{1},\left(A_{0}, A_{1}\right)_{1 / 2, r}\right)_{\eta, q} \\
& =\left(\left(A_{1}, A_{0}\right)_{1, \infty},\left(A_{1}, A_{0}\right)_{1 / 2, r}\right)_{\eta, q} \cap\left(\left(A_{0}, A_{1}\right)_{1, \infty},\left(A_{0}, A_{1}\right)_{1 / 2, r}\right)_{\eta, q} \\
& =\left(A_{1}, A_{0}\right)_{\theta, q} \cap\left(A_{0}, A_{1}\right)_{\theta, q}, \quad \frac{1}{2} \leqslant \theta<1 .
\end{aligned}
$$

Сопоставляя (13) и (20), приходим к (16). Докажем (17). Пусть $\frac{1}{2} \leqslant \theta<1$, $1<q<\infty, 1 / q+1 / q^{\prime}=1$. Тогда

$$
\begin{aligned}
& \left(A_{0}, A_{1}\right)_{\theta, q}+\left(A_{1}, A_{0}\right)_{\theta, q}=\left(\left(A_{0}, A_{1}\right)_{\theta, q}^{*} \cap\left(A_{1}, A_{0}\right)_{\theta, q}^{*}\right)^{*} \\
& \quad=\left(\left(A_{0}^{*}, A_{1}^{*}\right)_{\theta, q^{\prime}} \cap\left(A_{1}^{*}, A_{0}^{*}\right)_{\theta, q^{\prime}}\right)^{*}=\left(\left(\Sigma\left(\vec{A}^{*}\right), \Delta\left(\vec{A}^{*}\right)\right)_{\theta, q^{\prime}}\right)^{*} \\
& \quad=\left(\left(A_{0} \cap A_{1}\right)^{*},\left(A_{0}+A_{1}\right)^{*}\right)_{\theta, q^{\prime}}^{*}=(\Delta(\vec{A}), \Sigma(\vec{A}))_{\theta, q}=(\Sigma, \Delta)_{1-\theta, q} .
\end{aligned}
$$

Первое равенство написано на основании (11). Второе на основании теоремы 3.7.1 из [3]. Третье на основании (16), примененного к паре $\left\{A_{0}^{*}, A_{1}^{*}\right\}$. Четвертое на основании теоремы 2.7.1 из [3]. Пятое на основании теоремы 3.7.1 из [3] и рефлексивности пространств $\Sigma, \Delta$. Наконец, шестое равенство написано на основании теоремы 3.4.1 из [3]. Теорема доказана.

\section{§3. Применение к пространствам типа Соболева-Лиувилля и Никольского-Бесова}

Обозначим через $G^{+}$множество положительных функций $\nu(\xi) \in C^{\infty}\left(\mathbb{R}_{n}\right)$ таких, что для любого мультииндекса $\gamma$ с компонентами из множества $\{0 ; 1\}$

$$
\left|\xi^{\gamma} D^{\gamma} \nu(\xi)\right| \leqslant c \nu(\xi), \quad \xi \in \mathbb{R}_{n}, \quad \prod_{i=1}^{n} \xi_{i} \neq 0 .
$$

Пространства функций с гладкостью, задаваемой с помощью такой функции $\nu(\xi)$, рассматривались в [7].

ОПРЕДЕЛЕНИЕ 3 . Пусть $1<p<\infty,-\infty<s<\infty, \nu(\xi) \in G^{+}$. Положим

$$
H_{p}^{s}(\nu)=\left\{f \in S^{\prime}:\|f\|_{H}=\left\|F^{-1}\left(\left(1+\nu^{2}\right)^{s / 2} F f\right)\right\|_{L_{p}\left(\mathbb{R}_{n}\right)}<\infty\right\}
$$


Для положительной функции $\nu(\xi)$ из $C\left(\mathbb{R}_{n}\right)$, удовлетворяющей оценке $(21)$ и бесконечно дифференцируемой вне координатных осей, пространство $H_{p}^{s}(\nu)$ понимается как пополнение $S$ по определенной вьше норме.

Обозначим через $G_{0}^{+}$множество положительных на $\mathbb{R}_{n} \backslash\{0\}$ функций $\mu(\xi) \in C^{\infty}\left(\mathbb{R}_{n} \backslash\{0\}\right)$, непрерывных в нуле, удовлетворяющих оценке (21) и таких, что

$$
\mu(0)=0, \quad \lim _{|\xi| \rightarrow+\infty} \mu(\xi)=+\infty .
$$

ОПРЕДЕЛЕНИЕ 4 . Пусть $\mu \in G_{0}^{+}$. Через $\Phi(\mu)$ обозначим множество систем функций $\left\{\varphi_{k}(\mu ; x)\right\}_{k=0}^{\infty}$, обладаюших следуюшими свойствами:

a) $\varphi_{k} \in S\left(\mathbb{R}_{n}\right),\left(F \varphi_{k}\right)(\xi) \geqslant 0, k=0,1, \ldots$;

б) $\operatorname{supp} F \varphi_{k} \subset\left\{\xi \in \mathbb{R}_{n}: 2^{k-1} \leqslant \mu(\xi) \leqslant 2^{k+1}\right\}, k=1,2, \ldots$, $\operatorname{supp} F \varphi_{0} \subset\left\{\xi \in \mathbb{R}_{n}: \mu(\xi) \leqslant 2\right\}$;

в) $\sum_{k=0}^{\infty}\left(F \varphi_{k}\right)(\xi) \equiv 1, \xi \in \mathbb{R}_{n}$;

г) $\left\|F \varphi_{k}\right\|_{M_{p}} \leqslant c, k=1,2, \ldots$

Пример системы из $\Phi(\mu)$ приведен в [8].

ОПРЕДЕЛЕнИЕ 5. Пусть $1<p<\infty, 1<q<\infty,-\infty<s<\infty, \mu \in G_{0}^{+}$, $\left\{\varphi_{k}\right\}_{k=0}^{\infty} \in \Phi(\mu)$. Положим

$$
B_{p, q}^{s}(\mu)=\left\{f \in S^{\prime}:\|f\|_{B}=\left(\sum_{k=0}^{\infty} 2^{k s q}\left\|f * \varphi_{k}\right\|_{L_{p}\left(\mathbb{R}_{n}\right)}^{q}\right)^{1 / q}<\infty\right\} .
$$

В определениях $3-5 S-$ пространство Шварца, $F$ - оператор преобразования Фурье, $M_{p}$-пространство мультипликаторов Фурье типа $(p ; p)$.

При конкретизации функций $\mu$ и $\nu$ из приведенных выше определений $3-5$ получаются классические (изотропные и анизотропные) пространства Соболева-Лиувилля и Никольского-Бесова.

Tеорема 5. Пусть $1<p<\infty, 1<q<\infty, \mu_{0}, \mu_{1} \in G_{0}^{+}$. Интерполяционные пары $\left\{B_{p, q}^{1}\left(\mu_{0}\right), B_{p, q}^{1}\left(\mu_{1}\right)\right\} u\left\{H_{p}^{1}\left(\nu_{0}\right), H_{p}^{1}\left(\nu_{1}\right)\right\}$ квазилинеаризуемы. Соответствующие операторы задаются соотношениями

$$
V_{0}(t) a=F^{-1}\left(\frac{t \nu_{1}}{\nu_{0}+t \nu_{1}} F a\right), \quad V_{1}(t) a=F^{-1}\left(\frac{\nu_{0}}{\nu_{0}+t \nu_{1}} F a\right)
$$

где $\nu_{i}=\left(1+\mu_{i}^{2}\right)^{1 / 2}, i=0,1, a \in B_{p, q}^{1}\left(\mu_{0}\right)+B_{p, q}^{1}\left(\mu_{1}\right)$ (соответственно, $a \in H_{p}^{1}\left(\nu_{0}\right)+H_{p}^{1}\left(\nu_{1}\right)$ для пары $H$-пространств).

ДокАЗАтЕльство. Докажем утверждение теоремы для пары пространств типа Никольского-Бесова. Для пар пространств типа Соболева-Лиувилля утверждение доказывается аналогично. Проверим вьполнение условий (1)-(5). Условие (1) очевидно. Все оценки (2)-(5) проверяются аналогично с помощью теоремы Лизоркина о мультипликаторах Фурье (см. [9] и оценку (21)) и соответствуюших свойств $B$-пространств (см. [10]).

Проверим, например, [3]. Для краткости записи положим

$$
B_{i}=B_{p, q}^{1}\left(\mu_{i}\right), \quad i=0,1 .
$$


Для $a \in B_{1}$ имеем

$$
\begin{aligned}
\left\|V_{0}(t) a\right\|_{B_{0}}^{q} & =\sum_{k=0}^{\infty} 2^{k q}\left\|F^{-1}\left(\frac{t \nu_{1}}{\nu_{0}+t \nu_{1}} F \varphi_{k}\left(\mu_{0}\right) F a\right)\right\|_{L_{p}}^{q} \\
& \leqslant c \sum_{k=0}^{\infty}\left\|F^{-1}\left(\frac{t \nu_{0} \nu_{1}}{\nu_{0}+t \nu_{1}} F \varphi_{k}\left(\mu_{1}\right) F a\right)\right\|_{L_{p}}^{q} \\
& \leqslant c^{\prime} t \sum_{k=0}^{\infty}\left\|F^{-1}\left(\nu_{1} F \varphi_{k}\left(\mu_{1}\right) F a\right)\right\|_{L_{p}}^{q} \leqslant c^{\prime \prime} t\|a\|_{B_{1}} .
\end{aligned}
$$

Использовали теорему Лизоркина из [9] (см. оценку (21)) и теоремы 4, 6 из [10]. Теорема доказана.

Пусть $K\left(t, a ; A_{0}, A_{1}\right)$ - функционал Петре (см., например, [3]).

Одним из основных следствий квазилинеаризуемости пары $\left\{A_{0}, A_{1}\right\}$ является соотношение

$$
K\left(t, a ; A_{0}, A_{1}\right) \sim\left\|V_{0}(t) a\right\|_{A_{0}}+t\left\|V_{1}(t) a\right\|_{A_{1}},
$$

которое немедленно следует из (1)-(5) (см. [4, лемма 1.8.4]). В нашем случае из (22), (23) получаем (см. также [10])

$$
K\left(t, a ; B_{0}, B_{1}\right) \sim\left\|F^{-1}\left(\frac{t \nu_{0} \nu_{1}}{\nu_{0}+t \nu_{1}} F a\right)\right\|_{B_{p, q}^{0}} .
$$

Из соотношения (24) можно вывести множество интересных следствий (например, в духе теорем 1.8.5, 1.12.1 из [4]). Используя интерполяционные результаты из [10], можно вывести отсюда новые определения $B$-пространств (в том числе и для классических пространств). Не вдаваясь в подробности, отметим лишь одно следствие соотношения (24).

Теорема 6. В предположениях теореми 5 справедливо следующее:

a) $H_{p}^{1}\left(\nu_{0}\right) \cap H_{p}^{1}\left(\nu_{1}\right)=H_{p}^{1}\left(\nu_{0}+\nu_{1}\right), B_{p, q}^{1}\left(\mu_{0}\right) \cap B_{p, q}^{1}\left(\mu_{1}\right)=B_{p, q}^{1}\left(\mu_{0}+\mu_{1}\right)$;

б) $H_{p}^{1}\left(\nu_{0}\right)+H_{p}^{1}\left(\nu_{1}\right)=H_{p}^{1}\left(\frac{\nu_{0} \nu_{1}}{\nu_{0}+\nu_{1}}\right), B_{p, q}^{1}\left(\mu_{0}\right)+B_{p, q}^{1}\left(\mu_{1}\right)=B_{p, q}^{1}\left(\frac{\mu_{0} \mu_{1}}{\mu_{0}+\mu_{1}}\right)$.

ДокАЗАТЕЛЬСтво. Утверждения а) следуют из теоремы Лизоркина из [9] и теорем вложения разных метрик из [11].

У тверждения б) немедленно следуют из (24) (и соответствующего аналога для $H$-пространств), теоремы 6 из [10] и очевидного факта (с соответствующим видоизменением для $H$-пространств) $K\left(1, a ; B_{0}, B_{1}\right)=\|a\|_{B_{0}+B_{1}}$. Теорема доказана.

Таким образом, множества $H$ - и $B$-пространств замкнуты по отношению к операциям суммы и пересечения. Однако теорема 6 показывает, что сумма даже классических анизотропных $H$ - или $B$-пространств (с разной анизотропией и не лежащих один в другом) сама уже не является анизотропным пространством. Более того, она не может быть порождена каким-либо полным многогранником (см. [8]).

Поскольку при $1<p, q<\infty, 1 / p+1 / p^{\prime}=1 / q+1 / q^{\prime}=1,-\infty<s<\infty, \nu \in G^{+}$, $\mu \in G_{0}^{+}$

$$
\left(H_{p}^{s}(\nu)\right)^{*}=H_{p^{\prime}}^{-s}(\nu), \quad\left(B_{p, q}^{s}(\mu)\right)^{*}=B_{p^{\prime}, q^{\prime}}^{-s}(\mu)
$$


и $S$ плотно в $H_{p}^{s}(\nu), B_{p, q}^{s}(\mu)$ (доказательства этих утверждений аналогичны доказательствам соответствующих теорем для классических $H$ - и $B$-пространств, cм. [3], [7], [10], [12]), то на основании теоремы 6 и теоремы 2.7 из [3] получаем, что

$$
\begin{array}{lll}
B_{p, q}^{1}\left(\mu_{0}\right) \cap B_{p, q}^{1}\left(\mu_{1}\right) & \text { плотно в } & B_{p, q}^{1}\left(\mu_{i}\right), \\
\left(B_{p, q}^{1}\left(\mu_{0}\right)\right)^{*} \cap\left(B_{p, q}^{1}\left(\mu_{1}\right)\right)^{*} & \text { плотно в } & B_{p^{\prime}, q^{\prime}}^{-1}\left(\mu_{i}\right),
\end{array}
$$

где $1<p, q<\infty, 1 / p+1 / p^{\prime}=1 / q+1 / q^{\prime}=1, \mu_{i} \in G_{0}^{+}, i=0,1$.

Аналогичные соотношения плотности верны и для $H$-пространств.

Из приведенных выше соображений и теоремы 5 заключаем, что все изложенное в $\S \S 1,2$ может быть применено к парам $\left\{H_{p}^{1}\left(\nu_{0}\right), H_{p}^{1}\left(\nu_{1}\right)\right\}$ и $\left\{B_{p, q}^{1}\left(\mu_{0}\right), B_{p, q}^{1}\left(\mu_{1}\right)\right\}$.

\section{Список литературы}

1. Aronszajn N., Gagliardo E. Interpolation spaces and interpolation methods // Ann. Mat. Pura Appl. (4). 1965. V. 68. P. 51-117.

2. Peetre J. Zur Interpolation von Operatorenräume // Arch. Math. (Basel). 1970. V. 21. P. 601-608.

3. Берг Й., Лёфстрём Й. Интерполяционные пространства. Введение. М.: Мир, 1980.

4. Трибель $X$. Теория интерполяции. Функциональные пространства. Диффференциальные операторы. М.: Мир, 1980.

5. Peetre J. Über den Durchschnitt von Interpolationsräumen // Arch. Math. (Basel). 1974. V. 25. P. 511-513.

6. Berens $H$. Interpolationsmethoden zur Behandlung von Approximationsprozessen auf Banachräumen // Lect. Notes in Math. V. 64, 1968.

7. Triebel H. General function spaces. III: Spaces $B_{p, q}^{g(x)}$ and $F_{p, q}^{g(x)}, 1<p<\infty$ : basic properties // Anal. Math. 1977. V. 3. P. 221-249.

8. Багдасарян А. Г. Об интерполяции и следах функций из некоторых анизотропных функциональных пространств // Изв. АН Арм. ССР. 1988. Т. 23. № 4. С. 353-365.

9. Лизоркин П.И. $\left(L_{p}, L_{q}\right)$-мультипликаторы интегралов Фурье // Докл. АН СССР. 1963. T. 152. C. $808-811$.

10. Багдасарян А. Г. Интерполяция некоторых функциональных пространств разной анизотропии // Изв. АН Арм. 1992. Т. 27. № 4. С. 49-52.

11. Багдасарян А. Г. Теоремы вложения для некоторых обобщенных функциональных пространств // Изв. АН Арм. 1994. Т. 29. №3. С. 1-10.

12. Волевич Л. Р., Панеях Б. П. Некоторые пространства обобщенных функций и теоремы вложения // УМН. 1965. Т. 20. №1. С. 3-74. 